\title{
FREQUÊNCIA DE ASC-US EM LAUDOS CITOPATOLÓGICOS ALTERADOS E NÃO ALTERADOS EM UM LABORATÓRIO DA REDE PRIVADA DE FORTALEZA, CEARÁ
}

\author{
Antonia Fatima Pinheiro IELPO ${ }^{1 *}$, Cinthia Gabrielle Celedônio SILVA ${ }^{2}$, Francisco Gilson MALAGUETA \\ JUNIOR $^{2}$, Renata Mirian Nunes ELEUTÉRIO', Charles Ielpo MOURÃO ${ }^{2}$
}

1 Faculdade Ateneu. Fortaleza, Ceará, Brasil.

2 Universidade Estadual do Ceará. Fortaleza, Ceará, Brasil.

*Autor para correspondência: fatima.dip@ hotmail.com

DOI: http://dx.doi.org/10.18571/acbm.183

\section{RESUMO}

O câncer de colo do útero é considerado um problema de saúde pública no Brasil e no mundo. Sua incidência vem regredindo nos últimos anos, porém, a taxa de mortalidade ainda permanece alta. A doença se caracteriza pelo desenvolvimento de tumores a partir de alterações celulares na cérvice uterina, que podem ter início com uma simples lesão de baixo grau, ou até mesmo progredir lentamente até atingir estágios mais avançados da doença, tais como as de caráter maligno. $\mathrm{O}$ trabalho teve como objetivo investigar a incidência de laudos com diagnóstico compatível ao ASC-US entre os laudos citológicos alterados e não alterados em um Laboratório da rede privada de Anatomia Patológica, localizado na cidade de Fortaleza, Ce. O estudo é do tipo descritivo, transversal, retrospectivo, onde foi avaliada a frequência de laudos citopatológicos com resultados equivalentes a ASC-US, usando como banco de dados os registros do laboratório privado durante os anos de 2016 e 2017. Os resultados obtidos: a frequência de exames citopatológicos com diagnóstico de ASC-US foi de 33,58\%, entre o total de exames alterados, e de 3,29 considerando o valor total de citologias analisadas. Tais resultados se assemelham aos encontrados em estudo realizado no Serviço Integrado Tecnológico em Citologia do INCA, onde dentre os exames com alterações, 44,7\% eram ASC-US e entre todos os exames processados apenas 2,59\% dos resultados se referiam ao ASC-US. Conclui-se que a prevalência de células escamosas atípicas de significado indeterminado (ASC-US), no laboratório, foi de 3,29\%, não ultrapassando os $5 \%$ recomendados no sistema Bethesda.

Palavras-Chave: Colo; Lesão; Bethesda; Câncer.

\begin{abstract}
The Cervical cancer is considered a public health problem in Brazil and the world. Its incidence has been declining in recent years, however, the mortality rate still remains high. The disease is characterized by the development of tumors from cellular changes in the uterine cervix, which can start with a simple low-grade lesion, or even progress slowly to reach more advanced stages of the disease, such as malignant ones. The objective of this study was to investigate the incidence of reports with a diagnosis compatible with the ASC-US between altered and unchanged cytology reports in a laboratory of the Private Network of Pathological Anatomy, located in the city of Fortaleza, Ce. The descriptive, cross-sectional, retrospective study was carried out, where the frequency of cytopathological reports with equivalent results to ASC-US was evaluated, using the records of the private laboratory during the years 2016 and 2017 as a database: frequency of cytopathological exams with a diagnosis of ASC-US was $33.58 \%$, among the total of altered exams, and of 3.29 considering the total number of cytologies analyzed. These results are similar to those found in a study carried out at the Integrated Technological Service in Cytology of INCA, where among the exams with changes, $44.7 \%$ were ASC-US and among all the exams processed
\end{abstract}


only $2.59 \%$ of the results referred to ASC -US. It was concluded that the prevalence of atypical squamous cells of undetermined significance (ASC-US) in the laboratory was $3.29 \%$, not exceeding $5 \%$ recommended in the Bethesda system.

Keywords: Colo; Lesion; Bethesda; Cancer.

\section{Introdução}

O câncer no colo uterino é atualmente o segundo de maior prevalência entre as mulheres no mundo, com alta incidência e alta taxa de mortalidade. A prevenção básica consiste em identificar lesões pré-neoplásicas do colo uterino com administração de tratamento eficaz, evitando a progressão dessas células anormais em formas mais graves da doença. A estimativa de novos casos de câncer do colo uterino para o ano de 2018 é de mais de 18 mil, representando 8,1\% das neoplasias em mulheres, atrás apenas do câncer de mama e intestino (colo e reto) (BEZERRA et al., 2005; BRASIL, 2018; LEMOS et al., 2017).

A cérvice é a quarta localização anatômica mais frequente de câncer nas mulheres. Essa alta incidência da neoplasia é responsável por $15 \%$ das ocorrências de tumores malignos no colo do útero na população feminina, segundo Frigato e Hoga (2003).

No Brasil, o câncer de colo de útero é considerado um problema de saúde pública e apresenta um alto índice da doença. Além disso, por se tratar de um país em desenvolvimento, uma grande parcela das mulheres, em especial as que apresentam baixas condições econômicas, e na maioria das vezes também menor acesso às medidas preventivas, favorecem o aumento dos índices de prevalência e mortalidade (CESARIN; PICCOLI, 2011).

Segundo informações do Ministério da Saúde - MS (BRASIL, 2002), a evolução de lesões no corpo do colo uterino pode resultar no surgimento de carcinomas, sendo hoje um dos cânceres de mais fácil detecção, e quando comparado aos outros tipos de câncer o que apresenta um dos mais altos potenciais de cura, tendo o seu apogeu em mulheres de 40 a 60 anos de idade.

Para uma lesão de colo uterino atingir o estágio de carcinoma, células normais passam por uma série de alterações, tornando-se células pré-cancerosas (SELLORS; SANKARANARAYANAN, 2004). Caso não ocorra ação terapêutica, as células pré-malignas evoluem e atingem o estágio de carcinoma, de caráter maligno, que além de comprometer o tecido local podem invadir tecidos circundantes. Contudo, o rastreamento do câncer no colo uterino tem como principal finalidade detectar e tratar de lesões pré-neoplásicas, permitindo dessa forma que a incidência e a mortalidade sejam controladas (MACEDO, 2011).

Essas alterações são nomeadas de Neoplasia Intraepitelial Cervical (NIC), que surgem devido à desorganização de camadas celulares epiteliais, podendo ainda atingir outros tecidos como o conjuntivo, sendo classificadas em diferentes estágios, de acordo com a sua gravidade (BRASIL, 2002). Para determinar esses diferentes estágios foram desenvolvidos vários sistemas de classificação, porém, o mais utilizado em todo o mundo é o Bethesda, desenvolvido pelo Instituto Nacional do Câncer dos Estados Unidos. Nesse sistema é possível encontrar nomenclatura para designar desde lesões de alto grau até aquelas de caráter indeterminado, como é o caso do ASCUS (células escamosas atípicas de significado indeterminado). Essas células são assim representadas por apresentarem anormalidades mais expressivas que as encontradas em alterações de caráter inflamatório, porém, não apresentam qualitativa ou quantitativamente alterações suficientes para o diagnóstico de uma lesão (FERNANDES et al., 2012).

Os fatores que exercem risco para o desenvolvimento da doença referem-se à multiplicidade de parceiros, histórico de doenças sexualmente transmissíveis (DSTs), contágio com o Vírus do Papiloma Humano (HPV), a idade precoce da primeira relação sexual, a alimentação pobre e tabagismo, dentre outros (BRASIL, 2002). 
Para Cesarin e Piccoli (2011), no Brasil a principal forma de constatação da doença é a realização do exame citopatológico preventivo do câncer de colo de útero, conhecido popularmente como exame de Papanicolaou. Esse exame tem por objetivo detectar células anormais ou cancerosas auxiliando na tomada de medidas cabíveis para a regressão das lesões mediante tratamentos médicos.

Quanto às lesões do colo do útero, as que aparecem em maior frequência são as Atipias de Significado Indeterminado em células escamosas e glandulares, com resultados similares ao encontrado no trabalho de Dias, Tomazelli e Assis (2010). Essas lesões são caracterizadas por apresentarem anomalias nucleares mínimas e poucas divisões mitóticas, além de alterações que ocorrem basicamente nas camadas superiores do epitélio estratificado (SELLORS; SANKARANARAYANAN, 2004 e BRASIL, 2002).

Em 1988, um grupo multidisciplinar, envolvido em estudos sobre o câncer no colo do útero, patrocinados pelo Instituto Nacional do Câncer, na cidade de Bethesda, nos EUA, se reuniu para discutir a padronização dos laudos citopatológicos e propor terminologias que uniformizassem a comunicação entre os laboratórios e os profissionais envolvidos no processo. Dentre essas terminologias, surgiu o ASC-US que se refere, segundo Bethesda, à "alterações celulares reativas". Na época esse novo termo muito rapidamente ganhou aceitação em massa na maioria dos laboratórios clínicos. Em estudos realizados nesse mesmo período foi possível perceber que a taxa média de ASC-US nos laboratórios eram de 2,9\%, e apenas 10\% desses apresentavam taxa superior a 9\% (CONSOLARO; MARIA - ENGLER, 2016).

No Brasil, em 2002, um encontro promovido pelo Ministério da Saúde, através do Instituto Nacional do Câncer (INCA), especialistas passaram a recomendar a utilização do termo ASC-US para classificar atipias de significado indeterminado como alterações escamosas atípicas de significado indeterminado possivelmente não neoplásicas (FERNANDES et al., 2012). Porém, é importante ressaltar que estudos estimam que de 10 a $20 \%$ das mulheres com ASC-US tenham NIC II ou NIC III e, que uma em cada 1.000 possa ter câncer invasivo (CONSOLARO; MARIA - ENGLER, 2016).

Em levantamento realizado em 32 munícipios do estado do Rio de Janeiro entre os anos de 2007 e 2010, pelo SITEC/INCA, foi possível constatar que as colpocitologias com laudos alterados apresentaram um percentual de diagnósticos ASCUS equivalente a 44,7\%. Conforme informação descrita anteriormente, neste artigo, $49,23 \%$ dos exames com alterações apresentaram predominância nos diagnósticos de atipias em células escamosas, sendo a maioria do tipo ASCUS (FERNANDES et al., 2012).

Não muito diferente dos resultados supracitados, estudos realizados com 5.886 pacientes do sistema público de saúde de Guaporé, Vista Alegre do Prata e União da Serra (microrregião), Rio Grande do Sul, que realizaram exame entre novembro de 2011 e novembro de 2014, 269 $(4,57 \%)$ apresentaram alterações no exame citopatológico, desse total 96 (72,86\%) apresentaram resultados compatível com ASCUS (DALMOLIN et al., 2016). A Nomenclatura Brasileira para Laudos Cervicais e Condutas Preconizadas, cita que os ASCUS são as atipias mais comumente descritas nos laudos citopatológicos, com $80 \%$ dos relatos gerais dentre as lesões (BRASIL, 2006).

Um estudo realizado no munícipio de Maceió, no ano de 2007, previa investigar a presença de lesões de alto grau em mulheres com atipias de significado indeterminado. A análise dos resultados obtidos com a realização deste estudo possibilitou as seguintes conclusões: A prevalência de lesões intraepiteliais de alto grau em mulheres com diagnóstico colpocitológico de atipias de significado indeterminado (ASCUS) foi de 23,7\%, se mostrando dessa forma bastante elevada quando comparada a outras cidades brasileiras (COSTA; BARROS, 2011).

A escolha desta temática parte da ideia que está cada vez mais crescente a ocorrência de neoplasias malignas ligadas ao colo do útero na população feminina brasileira. Devido a ser uma doença de alta magnitude, que acomete alto percentual de mulheres entre 20 a 65 anos de idade, são imprescindíveis estudos sobre esse tema. Dessa forma, o monitoramento da incidência de 
lesões precursoras ao carcinoma no colo do útero pode levar a formulação de programas de prevenção e controle que busquem combater os fatores de risco. Tornam-se, então, indispensáveis; registros que apresentem dados atualizados e padronizados sobre a doença e que tais índices epidemiológicos sirvam como produto dos sistemas de vigilância.

\section{Metodologia}

\subsection{Tipo de Estudo}

Trata-se de um estudo descritivo, transversal, retrospectivo, onde se investiga a frequência de atipias de significado indeterminado do tipo ASC-US em exames citopatológicos ao longo de alguns meses dos anos de 2016 e 2017, no município de Fortaleza, Ceará, segundo dados levantados em um laboratório da rede privada.

\subsection{Coleta de Dados}

O presente artigo teve como base estudos bibliográficos e de documentação, por meio da análise de dados, oriundos de resultados de exames correspondentes à análise citológica cérvicovaginal de um laboratório clínico privado, localizado no município de Fortaleza / Ceará, que recebe amostras de pacientes de todas as 14 macrorregiões do Estado, a saber: Cariri, Centro Sul, Grande Fortaleza, Litoral Leste, Litoral Norte, Litoral Oeste/ Vale do Curu , Maciço de Baturité, Serra da Ibiapaba, Sertão Central, Sertão de Canindé, Sertão dos Crateús, Sertão dos Inhamuns, Sertão de Sobral e Vale do Jaguaribe.

Como requisitos éticos, foi resguardado o sigilo dos pacientes mantendo-se a identificação dos mesmos por um código numérico de uso exclusivo do laboratório, sendo disponibilizados apenas o código numérico, sexo e data de nascimento dos pacientes ora investigados. Para realização do levantamento dos dados, este estudo contou ainda com a autorização expressa e por escrito do responsável técnico do laboratório. Em adição, salienta-se que os resultados obtidos neste trabalho estão condicionados ao cumprimento dos princípios éticos contidos na Declaração de Helsinki (1964, reformulada em 1975, 1983, 1989, 1996 e 2000), da World Medical Association.

Para tanto, os laudos foram transcritos para um software de planilha eletrônica (tipo Microsoft Office Excel $^{\circledR}$ ), onde foram categorizados e tabulados de acordo com o Sistema de Bethesda e a Nomenclatura Brasileira para Laudos Cervicais e Condutas Preconizadas, conforme descrito pelo Ministério da Saúde e o Instituto Nacional do Câncer.

Os resultados foram analisados quanto à frequência de células escamosas atípicas de significado indeterminado (ASC-US) em laudos citopatológicos alterados e não alterados. Após tabulados, os resultados foram exibidos na forma de tabelas e gráficos e produzidos pelo software Microsoft Excel $^{\odot}$.

\section{Resultados e Discussão}

Este trabalho analisou 8.523 laudos ao longo de alguns meses dos anos de 2016 e 2017. Com base nos dados coletados foi possível chegar aos seguintes resultados: a análise de laudos em diferentes meses do ano de 2016 revelou que a frequência de diagnósticos ASC-US, nos exames processados durante esse mesmo período, foi de 2,23\%. Não muito diferente, em 2017, a análise apontou um percentual um pouco acima do valor encontrado em $2016 \mathrm{com}$ resultado equivalente a 4,38\%. Os dados encontrados nos meses de maio a dezembro de 2016 (exceto agosto) estão dispostos na tabela 1, ao passo que os dados obtidos nos exames realizados entre janeiro e agosto de 2017 (exceto abril e maio) estão dispostos na tabela 2. 
Tabela 1: Diagnóstico de ASC-US em diferentes meses do ano de 2016.

\begin{tabular}{|c|c|c|c|c|c|c|c|c|}
\hline $\begin{array}{c}\text { Mês/ } \\
\text { Diagnóstico }\end{array}$ & $\begin{array}{c}\mathrm{mai} / 16 \\
\mathrm{n}(\%)\end{array}$ & $\begin{array}{c}\text { jun/16 } \\
\text { n(\%) }\end{array}$ & $\begin{array}{l}\text { jul/16 } \\
\text { n(\%) }\end{array}$ & $\begin{array}{l}\text { set/16 } \\
\text { n(\%) }\end{array}$ & $\begin{array}{c}\text { out/16 } \\
\text { n(\%) }\end{array}$ & $\begin{array}{c}\text { nov/16 } \\
\text { n(\%) }\end{array}$ & $\begin{array}{c}\mathrm{dez} / 16 \\
\mathrm{n}(\%)\end{array}$ & $\begin{array}{l}\text { Total } \\
\mathrm{N}(\%) \\
\end{array}$ \\
\hline ASC-US & $\begin{array}{c}09 \\
(1,57)\end{array}$ & $\begin{array}{c}02 \\
(0,38)\end{array}$ & $\begin{array}{c}09 \\
(1,53)\end{array}$ & $\begin{array}{c}10 \\
(2,03)\end{array}$ & $\begin{array}{c}08 \\
(1,31)\end{array}$ & $\begin{array}{c}22 \\
(2,75)\end{array}$ & $\begin{array}{c}36 \\
(5,02)\end{array}$ & $\begin{array}{c}96 \\
(2,23)\end{array}$ \\
\hline Total & 572 & 526 & 589 & 492 & 609 & 799 & 716 & 4303 \\
\hline
\end{tabular}

Tabela 2: Diagnóstico de ASC-US em diferentes meses do ano 2017.

\begin{tabular}{cccccccc}
\hline $\begin{array}{c}\text { Mês/ } \\
\text { Diagnóstico }\end{array}$ & $\begin{array}{c}\mathbf{j a n} / \mathbf{1 7} \\
\mathbf{n}(\boldsymbol{\%})\end{array}$ & $\begin{array}{c}\mathbf{f e v / 1 7} \\
\mathbf{n}(\boldsymbol{\%})\end{array}$ & $\begin{array}{c}\mathbf{m a r} / \mathbf{1 7} \\
\mathbf{n}(\boldsymbol{\%})\end{array}$ & $\begin{array}{c}\mathbf{j u n} / \mathbf{1 7} \\
\mathbf{n}(\boldsymbol{\%})\end{array}$ & $\begin{array}{c}\mathbf{j u l} / \mathbf{1 7} \\
\mathbf{n}(\boldsymbol{\%})\end{array}$ & $\begin{array}{c}\text { ago/17 } \\
\mathbf{n}(\boldsymbol{\%})\end{array}$ & $\begin{array}{c}\text { Total } \\
\mathbf{n}(\%)\end{array}$ \\
\hline ASC-US & 14 & 15 & 41 & 35 & 60 & 20 & 185 \\
& $(2,16)$ & $(2,21)$ & $(5,14)$ & $(4,46)$ & $(8,82)$ & $(3,15)$ & $(4,38)$ \\
Total & 647 & 678 & 797 & 784 & 680 & 634 & 4.220 \\
\hline
\end{tabular}

Considerando os resultados dos exames citopatológicos que apresentaram laudos alterados em 2016, foi possível observar que de 217 casos, 96 (30, 68\%), tiveram seus resultados compatíveis com atipias de significado indeterminado, ASC-US. No ano de 2017, os valores encontrados não diferiram muito dos apresentados no ano de 2016, visto que, dos 339 casos de laudos alterados, 185 (35,30\%), correspondem ao ASC-US. Os dados encontrados nos meses de maio a dezembro de 2016 são apresentados na tabela 3, e relativos aos meses de janeiro a agosto de 2017 exibidos na tabela 4.

Tabela 3: Distribuição proporcional de diagnóstico ASC-US entre as citologias com laudos alterados, em diferentes meses de 2016.

\begin{tabular}{|c|c|c|c|c|c|c|c|c|}
\hline $\begin{array}{c}\text { Mês/ } \\
\text { Diagnóstico }\end{array}$ & $\begin{array}{c}\text { mai/16 } \\
n(\%)\end{array}$ & $\begin{array}{c}\text { jun/16 } \\
\text { n(\%) }\end{array}$ & $\begin{array}{l}\text { jul/16 } \\
\text { n(\%) }\end{array}$ & $\begin{array}{l}\text { set/16 } \\
\text { n(\%) }\end{array}$ & $\begin{array}{c}\text { out/16 } \\
\text { n(\%) }\end{array}$ & $\begin{array}{c}\text { nov/16 } \\
\text { n(\%) }\end{array}$ & $\begin{array}{c}\operatorname{dez} / 16 \\
n(\%)\end{array}$ & $\begin{array}{l}\text { Total } \\
\text { n(\%) }\end{array}$ \\
\hline ASC-US & $\begin{array}{c}09 \\
(29,03)\end{array}$ & $\begin{array}{c}02 \\
(20)\end{array}$ & $\begin{array}{c}09 \\
(37,5)\end{array}$ & $\begin{array}{c}10 \\
(27,78)\end{array}$ & $\begin{array}{c}08 \\
(18,60)\end{array}$ & $\begin{array}{c}22 \\
(29,72)\end{array}$ & $\begin{array}{c}36 \\
(37,90)\end{array}$ & $\begin{array}{c}96 \\
(30,68)\end{array}$ \\
\hline Outros & $\begin{array}{c}22 \\
(70,97)\end{array}$ & $\begin{array}{c}08 \\
(80)\end{array}$ & $\begin{array}{c}15 \\
(62,5)\end{array}$ & $\begin{array}{c}26 \\
(72,22)\end{array}$ & $\begin{array}{c}35 \\
(81,40)\end{array}$ & $\begin{array}{c}52 \\
(70,28)\end{array}$ & $\begin{array}{c}59 \\
(62,10)\end{array}$ & $\begin{array}{c}217 \\
(69,32)\end{array}$ \\
\hline Total & 31 & 10 & 24 & 36 & 43 & 74 & 95 & 313 \\
\hline
\end{tabular}

Nota: a categoria "outros" foi composta por células glandulares atípicas possivelmente não neoplásicas e quando não se pode excluir lesão de alto grau, adenocarcinoma, LSIL, HSIL, carcinoma epidermóide, e outras neoplasias. 
Tabela 4: Distribuição proporcional de diagnóstico ASC-US entre as citologias com laudos alterados, em diferentes meses de 2017.

\begin{tabular}{cccccccc}
\hline $\begin{array}{c}\text { Mês / } \\
\text { Diagnóstico }\end{array}$ & $\begin{array}{c}\mathrm{jan} / 17 \\
\mathrm{n}(\%)\end{array}$ & $\begin{array}{c}\mathrm{fev} / 17 \\
\mathrm{n}(\%)\end{array}$ & $\begin{array}{c}\mathrm{mar} / 17 \\
\mathrm{n}(\%)\end{array}$ & $\begin{array}{c}\mathrm{jun} / 17 \\
\mathrm{n}(\%)\end{array}$ & $\begin{array}{c}\mathrm{jul} / 17 \\
\mathrm{n}(\%)\end{array}$ & $\begin{array}{c}\mathrm{ago} / 17 \\
\mathrm{n}(\%)\end{array}$ & $\begin{array}{c}\text { Total } \\
\mathrm{n}(\%)\end{array}$ \\
\hline ASC-US & 14 & 15 & 41 & 35 & 60 & 20 & 185 \\
& $(31,11)$ & $(34,09)$ & $(32,54)$ & $(40,70)$ & $(37,03)$ & $(32,79)$ & $(35,30)$ \\
Outros & 31 & 29 & 85 & 51 & 102 & 41 & 339 \\
& $(68,89)$ & $(65,91)$ & $(67,46)$ & $(59,30)$ & $62,97)$ & $(67,21)$ & $(64,70)$ \\
Total & 45 & 44 & 126 & 86 & 162 & 61 & 524 \\
\hline
\end{tabular}

Nota: a categoria "outros" foi composta por células glandulares atípicas possivelmente não neoplásicas e quando não se pode excluir lesão de alto grau, adenocarcinoma, LSIL, HSIL, carcinoma epidermóide, e outras neoplasias.

No geral, considerando o acumulado dos dois anos, 2016 e 2017, das 837 citologias com resultados alterados, $281(33,58 \%)$ casos foram correspondentes a atipias de significado indeterminado (ASC-US), sendo a outra parcela, $556(66,42)$, da amostra representada, compatível com as demais atipias. Os dados mencionados estão descritos na figura 1.

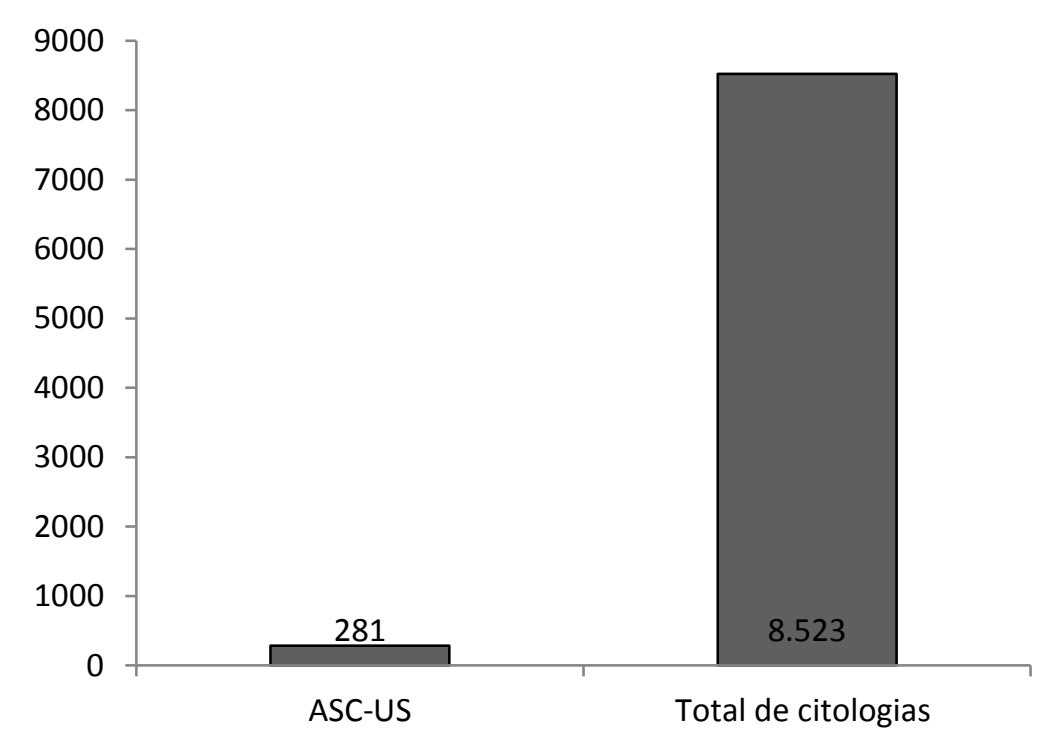

Figura 1: Distribuição geral do diagnóstico ASC-US entre todas as citologias que foram analisadas em meses dos anos 2016 e 2017.

Em se tratando da frequência do ASC-US no que se refere à análise do valor total de citologias analisadas nos dois anos, 8.523, o percentual de diagnóstico compatível com o ASCUS, foi equivalente a $3,29 \%$ da amostragem. Os dados apontados estão descritos na figura 2 . 


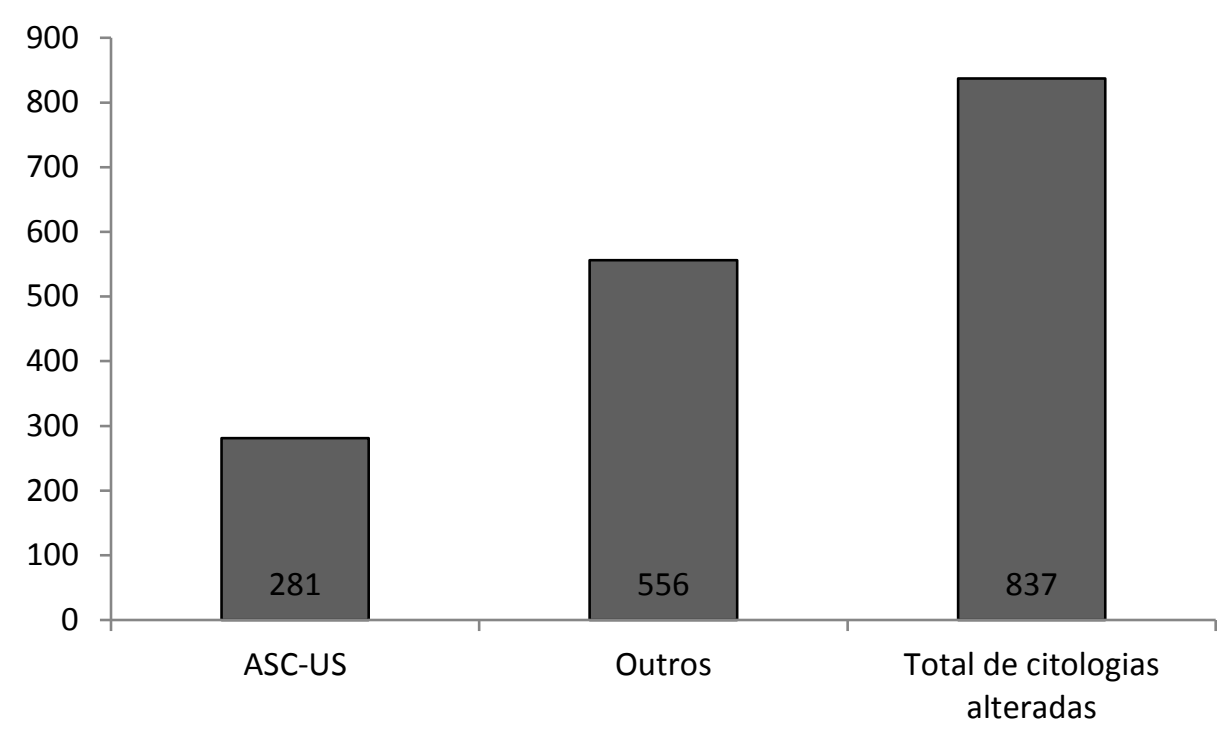

Figura 2: Distribuição geral do diagnóstico ASC-US entre as citologias com laudos alterados, de acordo com os meses analisados nos anos de 2016 e 2017 . Nota: a categoria "outros" foi composta por células glandulares atípicas possivelmente não neoplásicas e quando não se pode excluir lesão de alto grau, adenocarcinoma, LSIL, HSIL, carcinoma epidermóide, e outras neoplasias.

Resultados semelhantes podem ser encontrados em um estudo realizado por Fernandes et al., (2012), onde a prevalência do ASCUS nos exames com alterações foi de 49,23\%, sendo a maioria do tipo ASC-US (44,7\%).Ainda sobre esse levantamento, a análise dos relatórios de colpocitologia revelou que, entre todos os exames processados no período de 2007 a 2010, a frequência de diagnósticos ASC-US foi de 2,56\%. Não muito diferente disso, Dalmolin el at. (2016), constataram em suas pesquisas que das 269 (4,57\%) pacientes que apresentaram alterações no exame citopatológico, 96 (72,86\%), exibiram resultados compatíveis com ASC-US.

A importância de se realizar estudos que busquem apontar a incidência de diagnósticos citopatológicos equivalentes ao ASC-US deve principalmente a dois fatores: considerando que o principal objetivo do rastreio é identificar lesões precursoras do câncer do colo uterino, fazer um levantamento sobre a probabilidade de encontrar tais doenças quando uma mulher apresenta diagnóstico para ASC-US poderá ser uma forte ferramenta de prevenção. Além disso, essa mesma base de dados poderá oferecer subsídios para investigação posterior em pacientes que apresentem lesões cervicais invasivas (FERNANDES et al., 2012).

Ao mesmo tempo, muitos estudos revelam estatisticamente a probabilidade de pacientes que tiveram laudos de ASC-US desenvolverem NIC II ou NIC III. Para Consolaro e Maria-Engler (2016), essa possibilidade pode contemplar de $10 \%$ a 20\% dos casos. Todavia, Simsir et al., (2006) falam apenas da constância de 3\% de NICIII em pacientes diagnosticadas com ASC-US.

Em pesquisa realizada por Barros (2011), em um serviço público de referência foi constatado que dos 253 prontuários de mulheres com diagnóstico de ASC-US analisados a prevalência de lesões foi de $23,7 \%$, sendo $26,7 \%$ de baixo grau e $73,3 \%$ de alto grau. Com base nos dados apresentados é possível perceber o quanto é fundamental o desenvolvimento de estudos nessa área de interesse, no intuito de estarmos sempre buscando os melhores recursos na hora de promover decisões e recomendações (RUSSOMANO; MONTEIRO; MOUSINHO, 2008).

Além disso, certamente, o bom relacionamento e entrosamento entre os profissionais envolvidos nos processos que culminam no diagnóstico contribuirão para o sucesso do mesmo, e o consequente manejo correto das pacientes portadoras desse tipo de atipia (SEBASTIÃO et al., 2004). 


\section{Considerações Finais}

O percentual de atipias de significado indeterminado, no laboratório da rede privada em análise, encontra-se em total concordância com o recomendado pelo sistema Bethesda. Com base nos resultados encontrados é possível afirmar que a frequência do ASC-US não representa mais que 5\% do total de exames citopatológicos processados neste laboratório. Esses dados indicam a eficiência do controle de qualidade frente a todos os processos que culminam na qualidade do material e sua análise, conotando a excelência do laboratório para o processamento de citologias cérvico - vaginais.

\section{Referências}

BEZERRA, S. J. S.; GONÇALVES, P. C.; FRANCO. E. S.; PINHEIRO, A. K. B. Perfil de mulheres portadoras de lesões cervicais por HPV quanto aos fatores de risco para câncer de colo uterino. J bras doenças sex transm, v. 17, n. 2, p. 143-8, nov.2005.

BRASIL. Ministério da Saúde. Instituto Nacional de Câncer. Coordenação de Prevenção e Vigilância (Conprev). Falando sobre câncer do colo do útero. Rio de Janeiro: MS/INCA, 2002. $59 \mathrm{p}$.

BRASIL. Ministério da Saúde. Instituto Nacional de Câncer José Alencar Gomes da Silva. Coordenação de Prevenção e Vigilância [Internet]. Estimativa 2018: incidência de câncer no Brasil. Rio de Janeiro (RJ): INCA; 2018. [Citado 2018 ago 10]. Disponível em: http://www.inca.gov.br/estimativa/2018/casos-taxas-brasil.asp

CASARIN, M. R; PICCOLI, J. C. E. Educação em Saúde para Prevenção do Câncer de Colo do Útero em Mulheres do Município de Santo Ângelo/RS. Ciência \& Saúde Coletiva, v. 16, n. 9, p. 3925-3932, dez/jan. 2011.

CONSOLARO, M. E. L.; MARIA - ENGLER, S. S. (Org). Citologia clínica cérvico-vaginal: texto e atlas. São Paulo: Roca, 2016.

COSTA, R. F.; BARROS, S. M. O. Prevalência de lesões intraepiteliais em atipias de significado indeterminado em um serviço público de referência para neoplasias cervicais. Acta Paul Enferm, 2011; 24(3):400-6.

DALMOLIN, S. P., DEXHEIMER, G. M., DELVING, L. K. O. B. Mulheres com exames citopatológicos alterados: Avaliação do seguimento de acordo com as condutas preconizadas pelo Ministério da Saúde. Revista Brasileira de Análises Clínicas, 2016.

DIAS, M., B, K, JEANE G, TOMAZELLI M. A. Rastreamento do câncer de colo do útero no Brasil: análise de dados do Siscolo no período de 2002 a 2006. Epidemiologia e Serviços de Saúde, v. 19, n. 3, p. 293-306, jul/set 2010.

FERNANDES, F. et al., Diagnóstico Citopatológico de ASC-US e ASC-H no Serviço Integrado Tecnológico em Citologia do INCA. Revista Brasileira de Cancerologia, 2012; 58(3): 453-459. FRIGATO, S; HOGA, L. A. K. Assistência à mulher com câncer de colo uterino: o papel da enfermagem. Rev Bras Cancerol, v. 49, n. 4, p. 209-14, jul. 2003. 
FUNDAÇÃO OSWALDO CRUZ, Centro de Pesquisas Gonçalo Moniz, 2015. Disponível em: < http://www.arca.fiocruz.br/handle/icict/12238>. Acessado em: $12 \mathrm{~d}$ de fevereiro 2018.

LEMOS, A. R. M.; SILVA, M. S.; SEGATI, K. D. Lesão de alto grau e carcinoma escamoso: um estudo de prevalências em pacientes atendidas pelo Sistema Único de Saúde (SUS) na cidade de Anápolis, GO, Brasil. RBAC. v .49, n. 2, p. 152-7, 2017.

MACEDO, M. H. H. A. Atipias de significado indeterminado em células escamosas cervicais e diagnósticas histológicos na Secretaria de Estado de Saúde do Distrito Federal. 2011. Dissertação de mestrado. Universidade Estadual Paulista-UNESP, São Paulo, 2011.

SELLORS, JOHN W R. SANKARANARAYANAN, H.M.D. Colposcopia e tratamento da neoplasia intraepitelial cervical: manual para principiantes Washington, D.C. OPAS, (C) 2004. $120 \mathrm{p}$.

SIMSIR, A. et al., Effect of Bethesda 2001 on reporting of atypical squamous cells (ASC) with special emphasis on atypical squamous cells-cannot rule out high grade (ASC-H). Diagn Cytopathol. 2006;34(1):62-6.

RUSSOMANO, F; MONTEIR O, A. C. S; MOUSINHO, R. O. O diagnóstico citológico de células escamosas atípicas - uma avaliação crítica das recomendações diagnósticas. Rev Bras Ginecol Obstet. 2008; 30(11): 573-82.

SEBASTIÃO, A. P. M. et al. Estudo das atipias indeterminadas em relação à prevalência e ao percentual de discordância nos casos do Programa de Prevenção do Câncer Uterino do Paraná. J Bras Patol Med Lab. v. 40. n.6. p.431-8. Dezembro, 2004. 